We appreciate the response to this publication feature and welcome all contributions. Contributions may be sent to our Technical Editor Phil Oshel, oshellpe@cmich.edu

\section{A Note on Storing and Testing Gold Conjugates}

Jan Leunissen

Aurion, Costerweg, The Netherlands

leunissen@aurion.nl

Colloid and adsorption physics are a very complicated business and often have a lot of surprises. In 1991 Kramarcy and Sealock published a paper in JHC Vol. 39, No. 1,pp. 37-39, 1991: "Commercial Preparations of Colloidal Gold-Antibody Complexes Frequently Contain Free Active Antibody". Their data indicate that proteins adsorbed onto colloidal particles of $5 \mathrm{~nm}$ and larger can dissociate from the particle surface with time and that, at times even shortly after manufacturing, colloidal gold reagents may contain free binding molecules. This is not necessarily the result of bad manufacturing practice, as adsorption and desorption are in equilibrium at all times. Some proteins (there are even variations between antibodies from different animal species) are more liable to become dissociated than others, and the conditions of coupling play a role as well. If dissociation does occur, then older conjugates will progressively lose activity as a result and this will be partly because of the now less active reagent, and secondly because that reagent now has to compete with free (and thermodynamically more favorable) protein in solution.

Whether desorption will occur seems very much to be related to particle size. Ultra small conjugates prepared by adsorption have shown very consistent performance well beyond their shelf life. They do age well, and as much as this may be a surprise, it is a pleasant one (at least for the user!) and this illustrates that both adsorption based ultra small conjugates as well as antibodies can have a long and healthy life. Besides adsorption based production of gold reagents, the covalent binding principle is used with small organo-metallic gold particles, but I am not aware of this approach being used for larger particles.

How to test? What are sensible quality criteria? All producers of colloidal gold reagents (or any particle-based immuno reagents for that matter) will have their own in-house criteria. Some may use additional enhancement even with large 'colored' particles, while others may judge the performance exclusively based on the initial color.

So what does this mean in practice? With larger conjugates, one should be able to easily detect spots by eye when they contain between 1 and $10 \mathrm{ng}$ of specific protein in a dot-spot test under appropriate conditions that comply with the rules of affinity/avidity binding. Using enhancement, the levels may go down well into the picogram range.

Storing conjugates: Whenever one removes water from a hydrophilic structure that has partly hydrophobic qualities (as antibodies do), the risk of clustering based on hydrophobic interactions will definitely increase. This is especially true of conjugates built around large gold particles. London dispersion forces (e.g., van der Waals interactions) come into play as well. In case I would find myself at some point with a lot of conjugate left that was still reasonably, fresh, I would store it at $-20^{\circ} \mathrm{C}$ as a cryoprotected liquid (without freezing!), maybe using sucrose instead of glycerol, as glycerol may affect membranes. I have no solid data for this, but generally speaking desorption will be much reduced with lower temperatures, and should any clustering result from this treatment, a short spin of a ready-to-use diluted conjugate in a microfuge may remove such clusters. Some of the above is based on our in-house or personal experience and may not necessarily have been publicly documented.

Finally: in spite of all the criteria I mentioned, sometimes old conjugates do remarkably well, even though their performance in a dot-spot test would make one expect them to fail. I will say that if it is possible, it is always worth a try with a well know test specimen and primary antibody combination.

If anyone should be interested, we have a newsletter available by Peter van de Plas describing in detail how to perform simple tests that demonstrate activity and performance of gold conjugates, primary antibodies and enhancement reagents, even down to the level of antigen recognition.

\section{Cross-sectional TEM Sample Preparation for Nanowires or Porous Films Grown on a Substrate Chengyu Song \\ National Center for Electron Microscopy \\ Lawrence Berkeley National Laboratory csong@lbl.gov}

Nanowires or porous films grown on a substrate normally lack mechanical strength, and may be subject to damage during specimen preparation. When we made cross-sectional TEM specimen for this type of sample, we modified the traditional method by covering the sample with epoxy to improve the film strength, and applying single-section ion milling to protect the film from over-milling.

The sample surface is first covered with G1 epoxy. We choose G1 for this application because it is relatively thick and cures at low temperature. For samples with a dense-growth of nanowires or a thick porous film, a brief moment in vacuum helps to get rid of the air bubbles in the epoxy. The glue is cured at 100 degree $\mathrm{C}$ for 10 minutes, until its color turns to a reddish brown. To remove the excess glue and flatten the surface, the sample is then ground and polished until the glue is less than $0.1 \mathrm{~mm}$ thick.

Now the cross-sectional sample can be prepared using traditional methods. We glue the sample face-to-face to a piece of silicon wafer, using G1 or M-bond 610 epoxy. Additional pieces of silicon wafer can be added to both sides of the sample to make the stack 3 $\mathrm{mm}$ thick. Then it is sliced into $0.5 \mathrm{~mm}$ thick slices with a low speed diamond saw, drilled into a $3 \mathrm{~mm}$ diameter disc, and dimpled until the center part of the disc is around 10 microns thick.

To thin the sample to electron-transparency, a single-gun ion milling method is applied. The sample stage motion should be set to oscillate in a 60 degree arc. The ion beam should be only allowed to bombard the sample perpendicular to the substrate-to-film interface, at a low glancing angle of about 5 to 10 degrees. With such an arrangement, the substrate is acting as a shield for the film, protecting it from being over milled. 


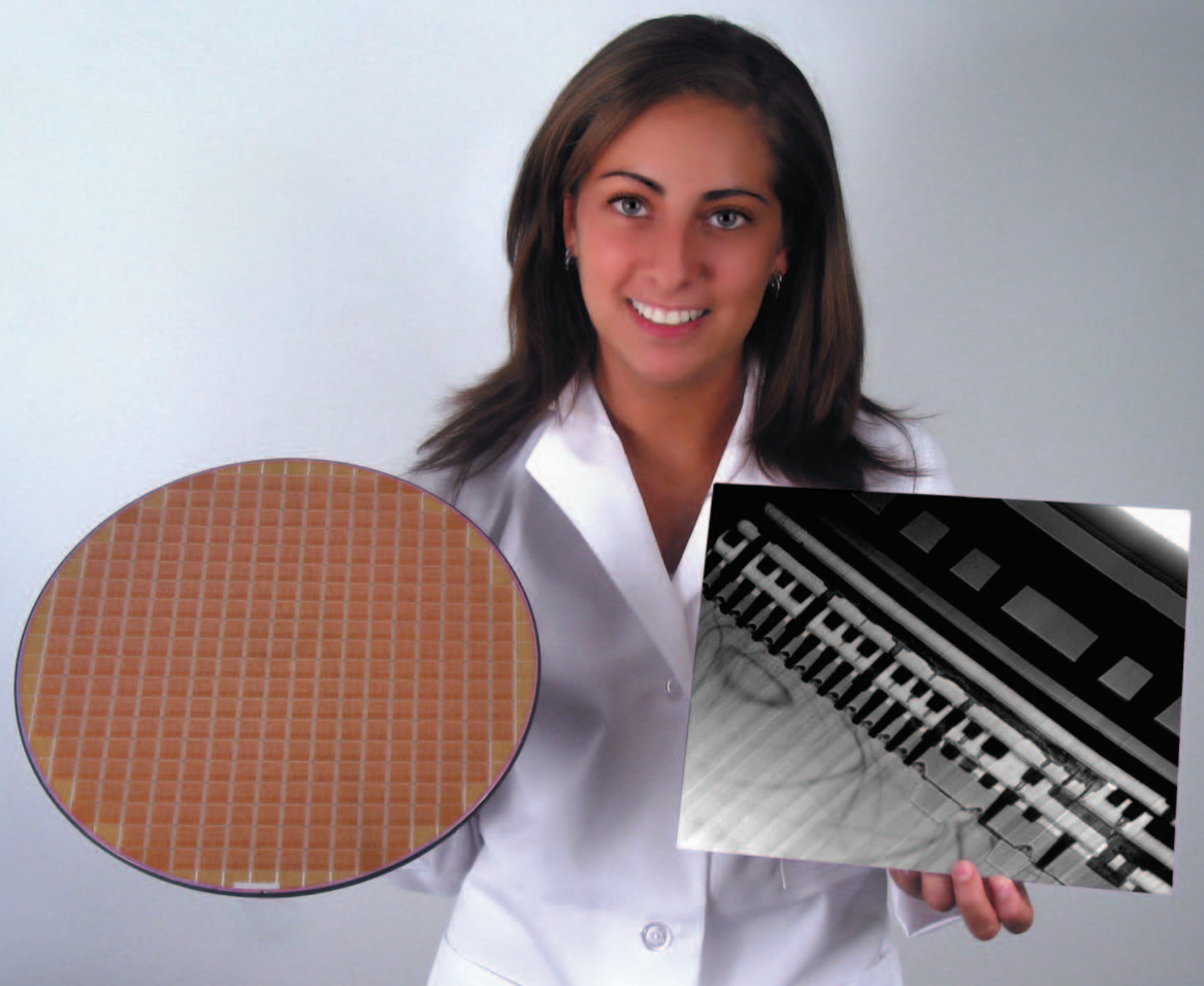

\section{Go from WAFER to RESULTS faster}

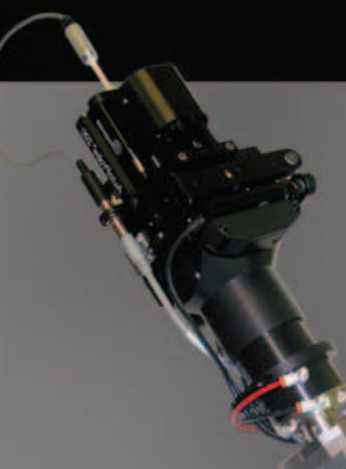

HIGH THROUGHPUT - AUTOMATION - HIGH SUCCESS RATE:

The AutoProbe 300 system for the FIB incorporates Omniprobe's Total Release lift-out method, 8th generation nanomanipulator, in-situ probe tip change and Short-Cut 'm technology for rapid and reliable TEM sample preparation.

For more information call 214.572 .6800 or visit our website. 